\title{
Magnetic anisotropy of epitaxial MgO/Fe/MgO films studied by network analyzer ferromagnetic resonance
}

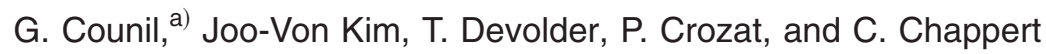 \\ Institut d'Electronique Fondamentale, Unité Mixte de Recherche (UMR) Centre National de la Recherche \\ Scientifique (CNRS) 8622, Université Paris-Sud, 91405 Orsay Cedex, France
}

\begin{abstract}
A. Cebollada
Instituto de Microelectrónica de Madrid IMM Centro Nacional de Microelectronica-Consejo Superior de Investigaciones (CNM-CSIC), Isaac Newton 8 Parque Tecnologico de Madrid (PTM), 28760 Tres Cantos (Madrid), Spain
\end{abstract}

(Received 30 November 2004; accepted 25 May 2005; published online 18 July 2005)

\begin{abstract}
The dynamic magnetic properties of $\mathrm{MgO} / \mathrm{Fe}$ epitaxial thin films are investigated through a network analyzer ferromagnetic resonance in the $0-26-\mathrm{GHz}$ range. The high-frequency response is measured for applied in-plane magnetic fields comparable to the fourfold magnetocrystalline anisotropy of the film, resulting in large-angle in-plane quasistatic motion of the magnetization. We show that the variation of the resonance frequency with both the amplitude and the direction of the external field is in good agreement with the ferromagnetic resonance equations, allowing precise determination of the anisotropy. (C) 2005 American Institute of Physics. [DOI: 10.1063/1.1968442]
\end{abstract}

\section{INTRODUCTION}

In magnetic recording, sensor devices, and magnetic read heads, the magnetic anisotropy plays a key role. Therefore, for both fundamental and technological reasons, the investigation of the magnetic anisotropy in ultrathin magnetic films is currently attracting significant attention. For example, much focus has been given to the epitaxial bcc crystalline Fe films recently because of their high structural quality, and as such, they provide a good model system in which the correlations between the magnetic anisotropy and physical structure can be probed with a number of different techniques. In earlier experiments, the transverse initial susceptibility ${ }^{1,2}$ (TBIS) technique has been used to determine the magnetic anisotropy of magnetic films in the quasistatic regime $(1 \mathrm{kHz})$.

At gigahertz frequencies, ferromagnetic resonance (FMR) has allowed the precise measurement of the static properties of magnetic films saturated by a large magnetic field. ${ }^{3-5}$ In such experiments, the resonance is probed by sweeping the applied field. The resonance fields provide a measurement of the effective field seen by the uniform precession mode that is excited by a uniform rf field. The effective field contains contributions from the external field, the demagnetizing field, and the magnetocrystalline energies. Standard FMR technique provides very high sensitivity due to the high-quality factor of the cavity at the working frequency, fixed by the geometry of the cavity. Then it has proved to be useful in providing accurate measures of the static properties of thin films, given by the magnetic anisotropy constants, and the dynamic properties, given by the linewidth $\Delta H$ of the resonance which provides information on relaxation processes

Recently, experiments using time-resolved magnetooptical kerr effect ${ }^{6}$ (MOKE) and pulsed inductive microwave

${ }^{a)}$ Electronic mail: counil@ief.u-psud.fr magnetometry ${ }^{7-9}$ (PIMM) has allowed to study the magnetic response of thin films in the time domain. With significant improvements in permeability measurement systems, ${ }^{10-14}$ the technique has enabled the precise characterization of magnetization dynamics in the frequency domain when attached to a network analyzer (NA-FMR). In NA-FMR experiments the resonance is probed by sweeping the frequency of the pumping field $h_{\mathrm{rf}}$. Here the inhomogeneity of the rf exciting field may induce distortion of the signal and needs to be taken into account when studying the evolution of both resonance frequency and linewidth $\Delta f$ with the external field,,${ }^{12,14,15}$ compared with the homogeneous exciting field created by the FMR cavity. Thus, the nonresonant NA-FMR system allows magnetization dynamics to be conveniently studied for a continuous range of applied fields and frequencies without any change of the experimental setup; thanks to the large $(>40 \mathrm{GHz})$ bandwidth of the system. Moreover, this technique provides both the real and imaginary parts of the complex susceptibility.

The anisotropic behavior at gigahertz frequencies of relaxation mechanisms such as the two magnon process are currently studied in Fe thin films, using FMR technique., ${ }^{4,16}$ The nonlinear evolution of the linewidth with frequency in the $0-20-\mathrm{GHz}$ range is one of the characteristics of this relaxation mechanism. However, at such frequencies, the resonance field is comparable to the in-plane magnetocrystalline anisotropy field and the FMR signal can become distorted. ${ }^{4}$ Indeed, the direction of the effective field varies throughout the measurement when the magnetization is not aligned with an easy direction of the magnetocrystalline anisotropy field. The measured linewidth $\Delta H$ thus reflects the effect of both the damping of the processional motion and the dragging of the magnetization behind the applied field. In NA-FMR, the equilibrium orientation of magnetization remains fixed as the frequency of the $\mathrm{rf}$ field is swept, provided that the $\mathrm{rf}$ field amplitude is small. 


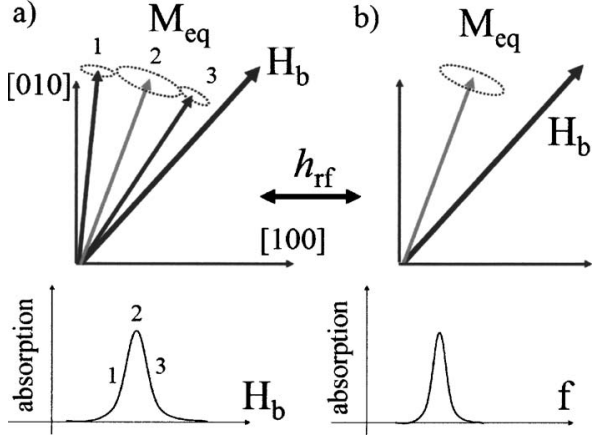

FIG. 1. Schematic picture of the resonance when measuring by FMR and NA-FMR. (a) In FMR measurements, the resonance is probed by scanning the external field $H_{b}$ around the resonant field for a given resonance frequency. During the scan, the static equilibrium magnetization $\mathbf{M}_{\mathrm{eq}}$ approaches toward the orientation of the applied field, and the effective field seen by the processing magnetization is changing. (b) In NA-FMR measurements, the external field value is fixed and the resonance is probed by scanning the frequency of the pumping field $h_{\mathrm{rf}}$.

In this paper we focus on the static properties of epitaxial $\mathrm{Fe} / \mathrm{MgO}$ thin films, which are highly attractive due to the good tunnel magnetoresistance (TMR) ratio of $\mathrm{Fe} / \mathrm{MgO} / \mathrm{Fe}$ multilayers. ${ }^{17}$ We demonstrate that the NA-FMR technique allows to study the static evolution of the magnetization at large angles with respect to the applied field by varying continuously its amplitude and direction. We show that the corresponding evolution of the resonance frequency can still be precisely fitted to the usual ferromagnetic resonance equations. Therefore, it allows accurate determination of both the magnitude and the direction of the magnetic anisotropy fields.

\section{EXPERIMENTAL SETUP AND SAMPLE PREPARATION}

Epitaxial $\mathrm{MgO}$ buffer layers, $12 \mathrm{~nm}$ thick, were grown at $450{ }^{\circ} \mathrm{C}$ by pulsed laser ablation on clean $\mathrm{MgO}$ surface (001). bcc Fe films with a thickness of $12 \mathrm{~nm}$ were subsequently deposited by triode sputtering at room temperature on the $\mathrm{MgO}$ buffer layer. ${ }^{18,19}$ As shown in previous studies, ${ }^{18}$ all lattice parameters are close to bulk values within $0.5 \%$ : there exists a small out-of-plane extension, which can be interpreted as a small Fe lattice accommodation due to the 3.8\% lattice mismatch with respect to $\mathrm{MgO}$ upon $45^{\circ}$ in-plane rotation. The epitaxial relations are then $\mathrm{Fe}(001)[110] / \mathrm{MgO}$ (001) [100], and the [100] and [010] crystallographic axes of Fe were found to be the easy axes of the fourfold magnetocrystalline anisotropy. ${ }^{19}$ A 6 -nm-thick $\mathrm{MgO}$ capping layer was then deposited to prevent oxidization of the magnetic film. (Fig. 1.)

The NA-FMR technique involves a measurement of the impedance of a coplanar waveguide loaded by the magnetic sample. ${ }^{11,12}$ The harmonic voltage sent through the waveguide creates a pumping field $h_{\mathrm{rf}}$ perpendicular to the waveguide which excites the precessional motion of the magnetization. The motion of the magnetic moment creates a mean flux variation, which in proper conditions is equivalent to a change of the characteristic impedance of the line $\Delta Z \propto \chi(f)$, where $\chi(f)$ is the complex susceptibility of the magnetic film. The impedance is first recorded with a 0.1-T static field

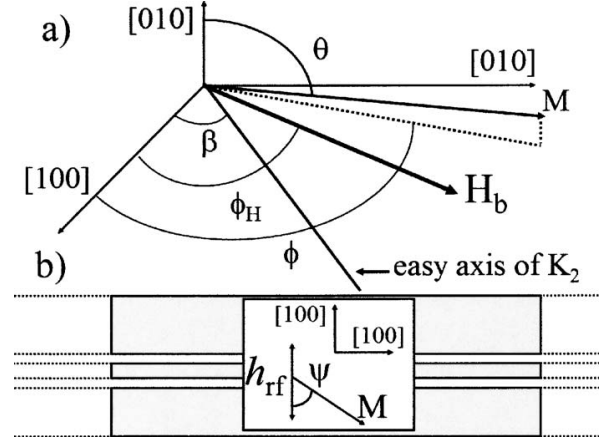

FIG. 2. (a) Coordinate system to describe the direction of the magnetization and the magnetic anisotropy. (b) Schematic description of the experimental setup. The magnetic sample is deposited on the coplanar waveguide. $\psi$ denotes the angle between the magnetization and the exciting field $h_{\mathrm{rf}}$ created by the waveguide.

applied parallel to the pumping field. For a pumping field $h_{\mathrm{rf}}$ with small amplitude, no spin waves are generated in this configuration. We then record the additional magnetic response with the static field applied in different directions and deduce the magnetic part of the response by subtracting the two recorded signals.

Here we use a small $\mathrm{rf}$ pumping field $\mu_{0} h_{\mathrm{rf}} \simeq 0.1 \mathrm{mT}$ to ensure that we remain in the linear regime. The extracted dynamic susceptibility of the magnetic film is well fitted to a Lorentzian profile, in good agreement with the solution of the linearized Gilbert equation. The imaginary part of the susceptibility $\chi^{\prime \prime}(f)$ is then given by

$$
\chi^{\prime \prime}(f)=\frac{A}{1+\left(\frac{f-f_{\mathrm{FMR}}}{\Delta f / 2}\right)^{2}} .
$$

This allows to extract the maximum amplitude $A$, the resonance frequency $f_{\mathrm{FMR}}$, and the linewidth $\Delta f$ of the resonance.

\section{THEORETICAL BACKGROUND}

Let us consider a system with both an in-plane fourfold anisotropy $K_{4}$ and an in-plane twofold anisotropy $K_{2}$. We define [100] and [010] as the directions of the fourfold anisotropy axes. We use $\beta, \phi_{H}$, and $\phi$ to denote, respectively, the angles between the [100] easy axis and the uniaxial anisotropy easy axis, external applied field, and magnetization, as illustrated in Fig. 2. The magnetic energy density is given by

$$
\begin{aligned}
E= & -\mu_{0} H_{b} M_{S} \sin \theta \cos \left(\phi_{H}-\phi\right)+\frac{1}{2} \mu_{0} M_{S}^{2} \cos ^{2} \theta \\
& +\frac{1}{4} K_{4}\left(\sin ^{2} 2 \theta+\sin ^{4} \theta \sin ^{2} 2 \phi\right) \\
& -K_{2} \sin ^{2} \theta \cos ^{2}(\phi-\beta),
\end{aligned}
$$

where $\mu_{0}$ is the permeability of free space, $M_{S}$ is the saturation magnetization, and $\theta$ is the angle between the film normal and the magnetization. The first term describes the Zeeman energy and the second term the demagnetizing energy. The third and fourth terms are the usual descriptions of the fourfold and twofold magnetocrystalline anisotropy energies, respectively. The equilibrium position of the magnetization is given by 


$$
\left[\frac{\partial E}{\partial \theta}\right]_{\theta=\theta_{0}}=\left[\frac{\partial E}{\partial \phi}\right]_{\phi=\phi_{0}}=0 .
$$

The first condition gives $\theta_{0}=\pi / 2$, and the second condition gives the in-plane angle $\phi_{0}$ of the magnetization at its equilibrium position. The resonance frequency $f_{\mathrm{FMR}}$ is then given by

$$
f_{\mathrm{FMR}}^{2}=\left(\frac{\gamma_{0}}{2 \pi \mu_{0} M_{S}}\right)^{2}\left[\frac{\partial^{2} E}{\partial \theta^{2}} \frac{\partial^{2} E}{\partial \phi^{2}}-\left(\frac{\partial E}{\partial \theta} \frac{\partial E}{\partial \phi}\right)^{2}\right]_{\theta_{0}, \phi_{0}},
$$

where $\gamma_{0}=\gamma \mu_{0}$ and $\gamma / 2 \pi=29.4 \mathrm{GHz} \mathrm{T}^{-1}$ being the gyromagnetic ratio. An analytical expression of Eq. (4) can be found when the external field $H_{b}$ is strong enough to align the equilibrium magnetization along its direction.

In what follows, we will consider that the twofold anisotropy is much smaller than the fourfold anisotropy, that is, $K_{2} \ll K_{4}$. For $H_{b}$ applied along one of the fourfold anisotropy easy axes, the magnetization then lies along the direction of $H_{b}$ at any applied field. The resonance condition is then given by

$$
\begin{aligned}
f_{\mathrm{FMR}}^{2}= & \left(\frac{\gamma_{0}}{2 \pi}\right)^{2}\left[M_{S}+H_{b}+H_{4}+\left(H_{2} / 2\right)(1 \pm \cos 2 \beta)\right] \\
& \times\left[H_{b}+H_{4} \pm H_{2} \cos 2 \beta\right],
\end{aligned}
$$

where $H_{4}=2 K_{4} / \mu_{0} M_{S}$ and $H_{2}=2 K_{2} / \mu_{0} M_{S}$ are, respectively, the fourfold and twofold anisotropy fields corresponding to the fourfold and twofold anisotropy energies. The + and refer, respectively, to the [001] and [010] directions. When $H_{b}$ is applied along a hard direction, the magnetization lies along the applied field when $H_{b}>\left|H_{4}\right|+\left|H_{2}\right|$, and the resonance condition is

$$
\begin{aligned}
f_{\mathrm{FMR}}^{2}= & \left(\frac{\gamma_{0}}{2 \pi}\right)^{2}\left[M_{S}+H_{b}+\left(H_{4} / 2\right)+\left(H_{2} / 2\right)(1 \pm \sin 2 \beta)\right] \\
& \times\left[H_{b}-H_{4} \pm H_{2} \sin 2 \beta\right]
\end{aligned}
$$

where the + and - refer, respectively, to is the [110] and $[1 \overline{1} 0]$ directions.

\section{EXPERIMENTAL RESULTS}

In Fig. 3, we show the measured squared resonance frequency versus the applied field $H_{b}$, with $H_{b}$ along the easy direction [100] and the hard direction [110] of the fourfold anisotropy. To ensure that the magnetization of the sample is well saturated, we apply a static field which is always above the coercive field $\mu_{0} H_{c} \simeq 1 \mathrm{mT}$ measured by MOKE (Fig. 4).

\section{A. Easy-axis measurements}

When $H_{b}$ is applied along the [100] easy direction, the magnetization lies along the direction of $H_{b}$, and the squared resonance frequency $f_{\mathrm{FMR}}^{2}$ follows a linear behavior as described by Eq. (5), as $M_{S} \gg H_{4}, H_{2}$. Graphically, the slope of the linear fit shown in Fig. 3 gives $M_{S}+H_{4}$, neglecting the variation of $H_{b}$, and its intersection with the field axis provides the anisotropy field $H_{4} \pm H_{2} \cos (2 \beta)$. A fit of the experimental data with Eq. (5) on both [100] and [010] easydirection measurements give the values of $M_{S}$ and

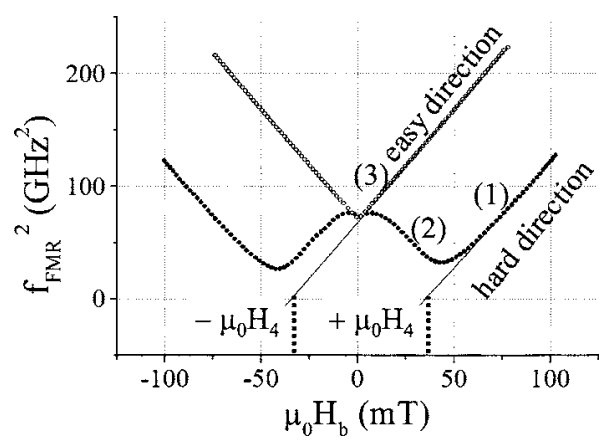

FIG. 3. Squared resonance frequency vs applied field $H_{b}$, with $H_{b}$ along the easy direction [100] $(\bigcirc)$ and the hard direction [110] (O). In this case, for $H_{b} \geqslant H_{4}(1)$, the magnetization lies along $H_{b}$. For $H_{b} \leqslant H_{4}(2)$, the magnetization rotates reversibly toward an easy direction. For $H_{b} \simeq 0$ (3), the magnetization lies along an easy direction. The solid lines are the linear fits. For negligible uniaxial anisotropy $K_{2} \ll K_{4}$, the intersection of the linear fits with the field axis is $\pm \mu_{0} H_{4}$, and the slope gives $\mu_{0} M_{S}$.

$H_{4} \pm H_{2} \cos 2 \beta$. From both fits, we obtain accurate values of $M_{S}, H_{4}$, and $H_{2} \cos (2 \beta)$. As expected, $H_{2} / H_{4}<3 \%$.

This is consistent with previous experiments ${ }^{19,20}$ on the same system which show that, in addition to the fourfold magnetic anisotropy due to the bcc structure of Fe, an additional uniaxial anisotropy is found superimposed. At low applied fields, this is evidenced by the difference between the magnetic reversal processes observed with the external field applied along either the [100] or the [010] direction (Fig. 4). The hysteresis loop, measured by MOKE, is square for the external field applied along the [010] direction. For the external field along the [100] direction, the hysteresis loop clearly shows plateaus, which indicates that the magnetization rotates $90^{\circ}$ with respect to the [100] direction, therefore pointing along the [010] direction. This uniaxial anisotropy was ascribed to interfacial origin ${ }^{19}$ due to the presence of steps on the $\mathrm{MgO}$ surface.

In previous studies on similar $\mathrm{Fe} / \mathrm{MgO}$ thin films with fourfold anisotropy and a uniaxial anisotropy superimposed, the latter is very likely to be along an easy direction, that is, $\beta=0^{\circ}$ or $90^{\circ}$. Experimental results, reported in Table I, are in good agreement with previously obtained values in both quasistatic TBIS measurements ${ }^{2,3}$ and high-frequency FMR measurements. ${ }^{3}$

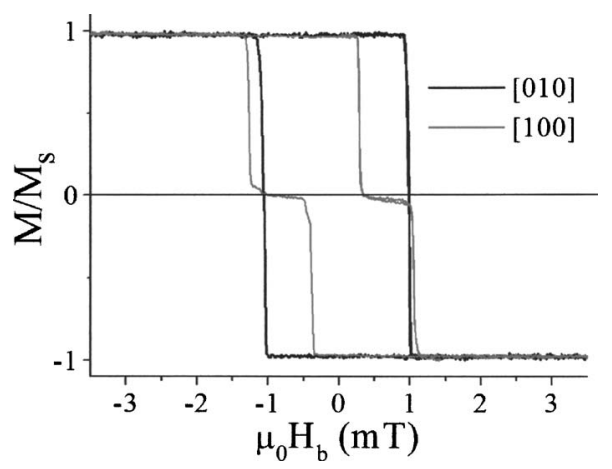

FIG. 4. Hysteresis loops performed by MOKE. When the external field is applied along the [100] direction, a two-step reversal is observed, which results from an additional uniaxial anisotropy axis lying along the [010] direction. 
TABLE I. Experimental values of $M_{S}, H_{4}$, and $H_{2}$.

\begin{tabular}{ccc}
\hline \hline $\begin{array}{c}\mu_{0} M_{S} \\
(\mathrm{~T})\end{array}$ & $\begin{array}{c}\mu_{0} H_{4} \\
(\mathrm{mT})\end{array}$ & $\begin{array}{c}\mu_{0} H_{2} \\
(\mathrm{mT})\end{array}$ \\
\hline $2.042 \pm 0.004$ & $37.97 \pm 0.04$ & $0.90 \pm 0.04$ \\
\hline \hline
\end{tabular}

We now study the gradual rotation of the magnetization when the field is applied along the hard axis and is gradually increased from 0 to $H_{b}>H_{4}$.

\section{B. Hard-axis measurements}

For $H_{b}$ applied along the [110] hard direction for instance, we observe three distinct parts in the static evolution of the magnetization. This is shown in Fig. 3: (1) At $H_{b}$ $>H_{4} \simeq 40 \mathrm{mT}$, the magnetization lies along the external field and $f_{\mathrm{FMR}}^{2}\left(H_{b}\right)$ follows a linear behavior as described by Eq. (6). (2) When $H_{b} \leqslant H_{4}$, energy minimization implies that the magnetization rotates reversibly toward an easy axis. In this case, the magnetization and the external field are no longer aligned and the FMR equations have to be solved numerically to account for the static rotation of the magnetization. (3) At zero applied field, the magnetization lies along an easy direction and the measured resonance frequency is then the same for both easy-and hard-direction measurements. The same dependence (not shown) is obtained for the [010] easy direction and $[1 \overline{10}]$ hard direction, which means that the system essentially exhibits a fourfold symmetry, i.e., $K_{2} \ll K_{4}$, as already deduced from easy-axis measurements.

To test the validity of the energy model when the angle $\left(\phi_{0}-\phi_{H}\right)$ between the magnetization and the external field is large, we use the values of $K_{4}$ and $K_{2}$ extracted from easyaxis measurements to evaluate numerically the resonance frequency and compare them to the experimental results (Fig. 5). Let us first detail the expected evolution of the magnetization. If $H_{b}$ is applied strictly along the hard-axis direction and $H_{2}=0$, we expect a soft mode to appear at $H_{b}=H_{4}$. If we take into account the measured uniaxial anisotropy, no soft mode is expected as the fourfold symmetry of the system is broken. In this case, the magnetization begins

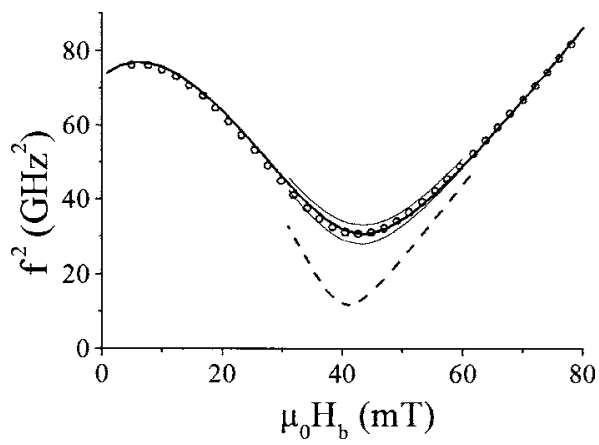

FIG. 5. Numerically calculated squared resonance frequencies (lines) and experimental data (open circles) for $H_{b}$ along [110] hard axis using the measured value of $K_{4}$ and $K_{2}$. The thick line is the calculated curve for $\phi_{H}=47.3^{\circ}$. The thin lines are the calculated curves for $\phi_{H}=47.1^{\circ}$ (lowest frequency) and $\phi_{H}=47.5^{\circ}$ (highest frequency), showing the $\pm 0.2^{\circ}$ precision on the misalignment. The dashed line is the calculated curve for $H_{b}$ strictly aligned with the hard direction.

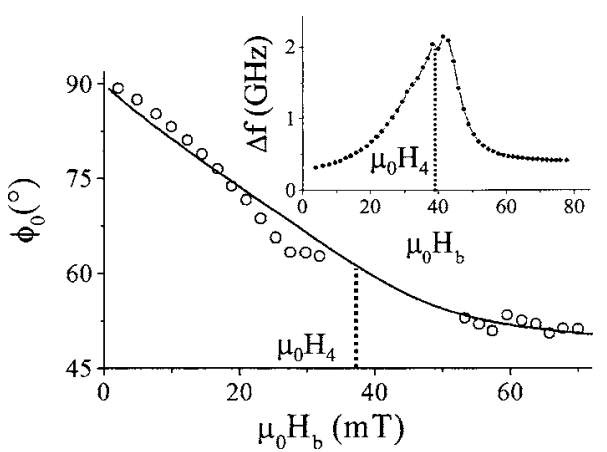

FIG. 6. Experimental data (circles) and theoretical result (solid line) for the angular position $\phi_{0}$ of the equilibrium magnetization $\mathbf{M}_{\mathrm{eq}}$ vs the angular position $\phi_{H}$ of the bias field $H_{b}$. No experimental data could be extracted at $H_{b} \simeq H_{4}$ due to the large increase of the inhomogeneous linewidth (see inset).

to rotate toward an easy axis at $H_{b}>H_{4}$, and a minimum in the resonance frequency should be observed (see the dashed line in Fig. 5).

However, the measured minimum resonance frequency is still greater than the minimum calculated by FMR equations. To account for this discrepancy, we have considered a slight misalignment of the applied field $H_{b}$ with the hard direction to account for the experimental data. The angle that describes best the experimental data can be determined with good precision, as the minimum resonance frequency is strongly affected by small deviations between the applied field and the hard-axis direction. The angle is found to be $\phi_{H}=47.3^{\circ} \pm 0.2^{\circ}$, consistent with the alignment precision of the electromagnet that generates the field $H_{b}$. The agreement between the calculated and measured data, in the whole field and frequency range, shows, that the static behavior of the magnetization is well described by the theoretical energy model. The $\pm 0.2^{\circ}$ error bar is determined roughly by the two extreme values of the misalignment which present an acceptable value of minimum frequency (see Fig. 5).

\section{Direct measurement of the angular position}

We now show that the measurement procedure allows the angular position of the equilibrium magnetization to be measured directly. Indeed, the area under $\chi^{\prime \prime}(f)$ is proportional to the volume of probed magnetic material $V$. The maximum amplitude $A$ of the measured signal, obtained from a Lorentzian fit, is then proportional to $V /\left(f_{\mathrm{FMR}} \times \Delta f\right)$ as long as the magnetization at its equilibrium position lies perpendicular to the pumping field $h_{\mathrm{rf}}$. However, the efficiency of the pumping field $h_{\mathrm{rf}}$ is modulated by a $\sin ^{2} \psi$ factor, ${ }^{12}$ where $\psi$ is the angle between the magnetization and the direction of $h_{\mathrm{rf}}$ [Fig. 2(b)]. We thus measure the pumping efficiency by looking at the evolution of the relative amplitude of the signal. This provides $\sin ^{2} \psi$, which is the angular position of the magnetization. In Fig. 6, we show that the experimental data are consistent with the theoretical angular position calculated from Eq. (3).

However, in the region $H_{b} \simeq H_{4}$ the linewidth dramatically increases due to the typical $1^{\circ}$ angular dispersion of the fourfold anisotropy. ${ }^{19}$ Indeed, we have shown that a slight change in the static parameters strongly changes the reso- 


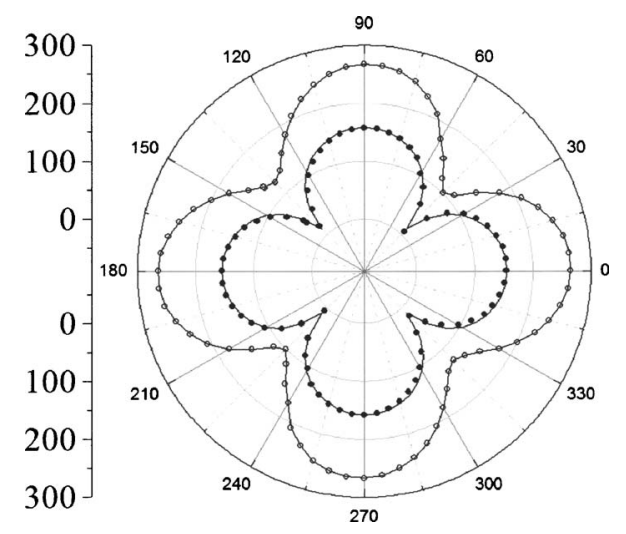

FIG. 7. Angular dependence of the (squared) resonance frequency at a strong external in-plane field $\mu_{0} H_{b}=100 \mathrm{mT}(\bigcirc)$. The solid line is a fit obtained from the analytical solution of FMR equations. At $\mu_{0} H_{b}=40 \mathrm{mT}$ (O), experimental data are well reproduced by FMR equations numerically solved, with values obtained from the fitting procedure at $100 \mathrm{mT}$.

nance frequency at $H_{b} \simeq H_{4}$. The inhomogeneous linewidth broadening is then maximum at such static field. As a consequence, the resonance profile is no longer Lorentzian, which renders the fitting procedure invalid. The overall linewidth, i.e., the peak-to-peak linewidth taken from the real part of the susceptibility, ${ }^{12}$ reaches $2 \mathrm{GHz}$ at $H_{b} \simeq H_{4}$ (see inset of Fig. 6). Therefore, the angle can not be measured precisely in this region. However, we have shown that NAFMR measurements allow us to gain access to the direction of the magnetization. Compared to MOKE measurements, this vectorial characterization of the magnetic reversal process is of great interest, as we will show in Sec. IV D.

\section{Polar measurements}

We have seen that the small misalignments between the applied field and the anisotropy hard axis can lead to large variations in the resonance response. To avoid such difficulties, one can also perform similar measurements with fixed field magnitude but varying direction of the applied field $\phi_{H}$. In this method, the small misalignment is no longer a source of error, as it is now a fitting parameter. Moreover, the angular dependence of the resonance frequency provides a simple picture of the anisotropy symmetry, as well as the value of the anisotropy constants which may be extracted from angular-dependent FMR equations. In Fig. 7, we present experimental results at $\mu_{0} H_{b}=40 \mathrm{mT}$ and $\mu_{0} H_{b}$ $=100 \mathrm{mT}$. Note that, for each orientation of the static field, the 100-mT field was applied first in order to avoid hysteretic behavior of the static magnetization. We first note that, at any applied field, the anisotropy of the magnetic film shows a clear fourfold symmetry. Let us assume that at $\mu_{0} H_{b}$ $=100 \mathrm{mT}$ the equilibrium magnetization and the external field are aligned. The FMR frequency can thus be calculated analytically from Eqs. (3) and (4). A fit from the analytical solution provides the magnetic parameters reported in Table II, consistent with the results of Table I.

However, this polar fitting procedure is less accurate than the fitting procedure performed on easy-axis measurements, reflected by greater error bars. Indeed, it can be seen in Fig. 8 that the equilibrium magnetization is not always
TABLE II. Experimental values of $M_{S}, H_{4}, H_{2}$, and $\beta$ obtained by polar fit.

\begin{tabular}{ccccc}
\hline \hline $\begin{array}{c}H_{b} \\
(\mathrm{mT})\end{array}$ & $\begin{array}{c}\mu_{0} M_{S} \\
(\mathrm{~T})\end{array}$ & $\begin{array}{c}\mu_{0} H_{4} \\
(\mathrm{mT})\end{array}$ & $\begin{array}{c}\mu_{0} H_{2} \\
(\mathrm{mT})\end{array}$ & $\beta\left(^{\circ}\right)$ \\
\hline 100 & $2.05 \pm 0.02$ & $37.5 \pm 1$ & $1.7 \pm 1$ & $77 \pm 17$ \\
\hline \hline
\end{tabular}

perfectly aligned with the bias field. The angle $\phi_{0}$ was measured using the procedure described in Sec. IV C. Note that the experimental misalignments are in perfect agreement with the theoretical angular position calculated from Eq. (3). In this case, the analytical solution used for the fitting procedure is not strictly valid, resulting in a less accurate determination of the magnetic anisotropy constants. We would like to emphasize that anisotropic contribution to the linewidth has been observed, but the inhomogeneous contribution already mentioned still needs to be discriminated from the intrinsic contribution such as the two-magnon process. ${ }^{4}$

At $\mu_{0} H_{b}=40 \mathrm{mT}$, the magnetization is generally not aligned with the applied field. In this case, a numerical fitting procedure is needed to extract the magnetic parameters. To check for consistency, we show that the model reproduces well the experimental data. Taking the values of magnetic parameters extracted from the previous fitting procedure, we show both the calculated data from FMR equations and the experimental data in Fig. 7. We see that FMR equations still describe well the magnetization dynamics at any angular position and at any magnitude of the external applied field. The energy model is then valid at any applied fields and at any angle, indicating that the magnetization is fairly homogeneous inside the sample.

\section{CONCLUSION}

We have shown that NA-FMR has provided further insight into the broadband response of anisotropic magnetic materials. In our epitaxial $\mathrm{Fe} / \mathrm{MgO}$ system, the applied and magnetocrystalline anisotropy fields are of the same order of magnitude, and the angle between the magnetization and the applied field can vary over a large range. Nevertheless, we have shown that the technique allows the static properties to be determined unambiguously, irrespective of the direction of the equilibrium magnetization relative to the external bias field. The theoretical energy model used to describe the magnetic anisotropy fits well with the experimental data in the

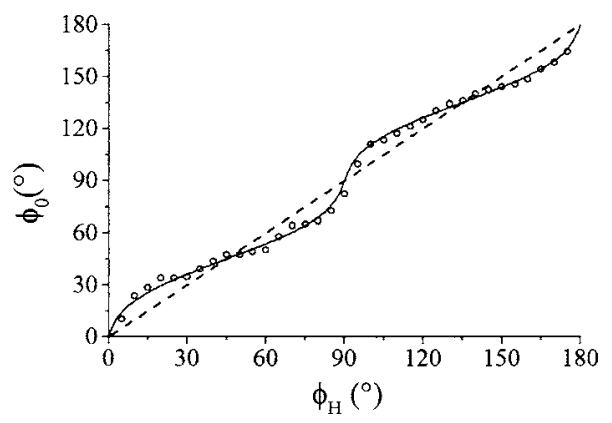

FIG. 8. Experimental data (circles) and theory (line) for the angular position $\phi_{0}$ of the equilibrium magnetization $\mathbf{M}_{\mathrm{eq}}$ as a function of the angular position $\phi_{H}$ of the bias field $H_{b}$. The dashed line represents the state for which the magnetization would be perfectly aligned with the bias field. 
whole $0-26-\mathrm{GHz}$ dynamic range, which allows us to determine the anisotropy constants accurately. We have shown that this model can be precisely fitted to both static and dynamic behaviors of the magnetization, especially in the case when the magnetization is not aligned with the bias field.

Scanning the frequency instead of scanning the field avoids any dragging of the magnetization while probing the resonance. The frequency linewidth can thus be studied for all in-plane orientations of the magnetization, in the whole $0-20-\mathrm{GHz}$ frequency range. We are currently studying the anisotropic contribution to the linewidth observed during polar measurements, which opens the way to a better understanding of relaxation processes such as two-magnon scattering in Fe thin films. ${ }^{21-23}$

\section{ACKNOWLEDGMENTS}

This work was supported by the European Communities Human Potential program under Contract No. HRPN-CT2002-00318 ULTRASWITCH and by the Spanish Commission of Science and Technology. The authors would like to thank J. L. Costa-Krämer for his help with Kerr measurements and J. L. Menendez for helpful discussions.

${ }^{1}$ F. Cebollada, A. Hernando-Mañeru, A. Hernando, C. Martínez-Boubeta, A. Cebollada, and J. M. González, Phys. Rev. B 66, 174410 (2002).

${ }^{2}$ J. F. Calleja, J. L. Menéndez, A. Cebollada, and C. Contreras, Jpn. J. Appl. Phys., Part 1 40, 6829 (2001).

${ }^{3}$ R. Urban, G. Woltersdorf, and B. Heinrich, Phys. Rev. Lett. 87, 217204 (2001).

${ }^{4}$ G. Woltersdorf and B. Heinrich, Phys. Rev. B 69, 184417 (2004).
${ }^{5}$ D. J. Twisselmann and R. D. McMichael, J. Appl. Phys. 93, 6903 (2003). ${ }^{6}$ L. Lagae, R. Wirix-Speetjens, W. Eyckmans, S. Borghs, and J. De Boeck, J. Magn. Magn. Mater. 286, 291 (2005).

${ }^{7}$ T. J. Silva, C. S. Lee, T. M. Crawford, and C. T. Rogers, J. Appl. Phys. 85, 7849 (1999).

${ }^{8}$ T. M. Crawford, M. Covington, and G. J. Parker, Phys. Rev. B 67, 024411 (2003).

${ }^{9}$ M. Covington, T. M. Crawford, and G. J. Parker, Phys. Rev. Lett. 89, 237202 (2002)

${ }^{10}$ D. Pain, M. Ledieu, O. Acher, A. L. Adenot, and F. Duverger, J. Appl. Phys. 85, 5151 (1999).

${ }^{11}$ G. Counil, J.-V. Kim, K. Shigeto, Y. Otani, T. Devolder, P. Crozat, H. Hurdequint, and C. Chappert, J. Magn. Magn. Mater. 272-276, 290 (2004).

${ }^{12}$ G. Counil, J.-V. Kim, K. Shigeto, Y. Otani, T. Devolder, and C. Chappert, J. Appl. Phys. 95, 5646 (2004).

${ }^{13}$ B. K. Kuanr, R. E. Camley, and Z. Celinski, J. Appl. Phys. 95, 6610 (2004).

${ }^{14}$ B. K. Kuanr, R. E. Camley, and Z. Celinski, J. Magn. Magn. Mater. 286, 276 (2005)

${ }^{15}$ M. L. Schneider, A. B. Kos, and T. J. Silva, Appl. Phys. Lett. 85, 254 (2004).

${ }^{16}$ J. Lindner et al., Phys. Rev. B 68, 060102(R) (2003).

${ }^{17}$ S. Yuasa, A. Fukushima, T. Nagahama, K. Ando, and Y. Suzuki, Jpn. J. Appl. Phys., Part 2 43, L588 (2004).

${ }^{18}$ C. Martínez Boubeta, E. Navarro, A. Cebollada, F. Briones, F. Peiró, and A. Cornet, J. Cryst. Growth 226, 223 (2001).

${ }^{19}$ C. Martínez Boubeta, J. L. Costa-Krämer, and A. Cebollada, J. Phys.: Condens. Matter 15, R1123-R1167 (2003).

${ }^{20}$ R. P. Cowburn, S. J. Gray, J. Ferré, J. A. C. Bland, and J. Miltat, J. Appl. Phys. 78, 7210 (1995)

${ }^{21}$ R. Arias and D. L. Mills, Phys. Rev. B 60, 7395 (1999).

${ }^{22}$ B. K. Kuanr, R. E. Camley, and Z. Celinski, J. Appl. Phys. 93, 7723 (2003).

${ }^{23}$ S. M. Rezende, A. Azevedo, M. A. Lucena, and F. M. Aguiar, Phys. Rev. B 63, 214416 (2001). 